\title{
Perceptions de la compétence à communiquer oralement du personnel d'un service de garde en milieu scolaire : le cas d'une école montréalaise
}

\author{
CHRISTIAN DUMAIS \\ Université du Québec à Trois-Rivières \\ EMMANUELLE SOUCY \\ Université du Québec en Outaouais
}

\section{Résumé}

En tant que modèles linguistiques, tous les acteurs de l'école ont un rôle à jouer dans le développement de la compétence à communiquer oralement des élèves, une compétence de premier plan en littératie. C'est notamment le cas du personnel des services de garde en milieu scolaire (SGMS) (Cloutier, 2012; Berthiaume, 2016). Actuellement, nous en savons très peu sur la compétence à communiquer oralement de ce personnel et sur les perceptions de ce dernier quant à l'utilisation de la langue orale en milieu scolaire (Bergeron, Dumais, Harvey \& Nolin, 2015). Afin d'explorer cette zone d'ombre de la recherche, nous avons mené une recherche exploratoire qui avait pour objectif de décrire les perceptions du personnel d'un SGMS d'une école primaire montréalaise quant à sa compétence à communiquer oralement.

\section{Mots-clés}

communication orale, compétence, service de garde en milieu scolaire, éducateur, surveillant du diner

\section{Introduction}

Au Québec, depuis la fin des années 1970, on retrouve dans la plupart des écoles primaires un service de garde ${ }^{1}$. Ce service de garde en milieu scolaire (SGMS) est un service éducatif complémentaire à ceux fournis par l'école et il est administré par cette dernière ainsi que par la commission scolaire (Giguère \& Desrosiers, 2010). Le SGMS est offert aux enfants de 4 à 12 ans. Des éducatrices et des éducateurs, qui ont habituellement un diplôme d'études collégiales en service de garde, sont responsables des enfants avant le début des classes, le midi et après la fin des classes (environ six heures par jour ${ }^{2}$ ) pour un ratio d'un adulte pour 20 enfants. Des surveillantes et surveillants du diner peuvent également être présents le midi. Les membres du personnel des SGMS, tant les éducatrices et éducateurs en services de garde que les surveillantes et surveillants du diner, accompagnent les enfants dans leur développement et «jouent un rôle de socialisation important auprès [d'eux], témoignant de l'extension du rôle social de l'école » (Caron, 2016, p. ii). Le personnel des SGMS participe donc au développement des compétences des enfants du préscolaire et du primaire, et il est un partenaire du personnel enseignant

\footnotetext{
${ }^{1}$ Selon le Conseil supérieur de l’éducation (2006), plus de 80\% des écoles primaires du Québec avaient un service de garde en milieu scolaire en 2004-2005.

${ }^{2}$ Lors des journées pédagogiques, le SGMS peut avoir une durée d’ouverture d'environ 11 heures (Berger, Héroux \& Shéridan, 2012).
} 
(Berger, Héroux \& Shéridan, 2012). En effet, le personnel des SGMS doit « veiller au bienêtre général des élèves et poursuivre, dans le cadre du projet éducatif de l'école, le développement global des élèves par l'élaboration d'activités tenant compte de leurs intérêts et de leurs besoins, en complémentarité aux services éducatifs de l'école » (Gouvernement du Québec, 2017, p. 2). Malgré ce rôle majeur auprès des enfants, nous en savons très peu sur les compétences de ce personnel, plus particulièrement en ce qui concerne la compétence à communiquer oralement, une compétence essentielle dans le cadre de cette profession (Bergeron et al., 2015; Berthiaume, 2016). Cette contribution fait état d'une recherche exploratoire menée auprès de 12 sujets d'un SGMS d'une école primaire montréalaise. Il est question de leurs perceptions quant à leur compétence à communiquer oralement, une compétence de premier plan en ce qui concerne la littératie (Dumais, 2015; Dumais, Soucy \& Plessis-Bélair, 2017).

\section{Problématique}

Malgré une croissance importante des SGMS au Québec - le nombre de SGMS a presque quadruplé entre 1986 et 2010 passant de 422 SGMS à 1581 SGMS (Association des services de garde en milieu scolaire du Québec, 2011) -, peu de chercheurs se sont intéressés au cours des dernières années à ce qui se fait au sein des SGMS, aux apports de ces derniers auprès des enfants ainsi qu'à la formation et aux compétences du personnel des SGMS. En effet, « sur le plan de la recherche, les services de garde en milieu scolaire ont été négligés » et « les effets de la fréquentation de ces services demeurent mal connus » (Giguère \& Desrosiers, 2010, p.16). De plus, « les études sur la formation du personnel des services de garde en milieu scolaire sont plus rares » (Conseil supérieur de l'éducation [CSÉ], 2012, p. 28).

Les quelques recherches qui ont été menées en lien avec les SGMS au cours des dernières années ont fait état de certains problèmes importants. Parmi ces derniers, on relève entre autres une fréquence peu élevée de rencontres entre le personnel des SGMS et le personnel enseignant pendant l'année scolaire (Tessier, Turcotte, Perreault, Beaudoin \& Berrigan, 2016). Certaines recherches (Levasseur \& Tardif, 2005; Chalifoux, MyreBisaillon \& Bergeron, 2014; Sorel Étienne, Lambert Deubelbeiss \& Rousseau, 2015) mentionnent même une absence de moments d'échanges sans la présence des enfants. De cette situation résulterait un manque d'informations partagées entre les enseignants et le personnel des SGMS (plan d'intervention associé à un élève, comportements inadéquats d'un enfant, problématiques particulières, mesures disciplinaires, etc.), ce qui occasionnerait une méconnaissance des réalités vécues au service de garde et en classe pour chacun (CSÉ, 2017). Cette situation rendrait « difficile la continuité éducative souhaitée auprès des enfants qui fréquentent les deux milieux » (Chalifoux et al., 2014, p. 45) et causerait un manque de cohésion entre le personnel des SGMS et les enseignants dans l'application des règles (Tessier et al., 2016). Un autre problème soulevé concerne les interventions à faire auprès des enfants plurilingues. "Le personnel des services de garde est parfois démuni devant les besoins de ces enfants et [...] il bénéficie rarement d'un soutien analogue à celui qui est offert en classe » (CSÉ, 2006, p. 46). La situation est similaire pour les enfants ayant des besoins particuliers (Conseil de la famille et de l'enfance, 2006). Enfin, un élément problématique relevé à quelques reprises dans les écrits concerne le partage des locaux entre le personnel enseignant et le personnel des SGMS, notamment en raison d'un manque de temps pour discuter des règles à suivre dans les 
locaux (Cayouette, 2017). Cette situation serait une source de conflits à l'intérieur des équipes-écoles (Berger et al., 2012). La communication semble être au centre de plusieurs de ces problèmes vécus dans les SGMS.

Le Conseil supérieur de l'éducation du Québec, qui a pour mandat de conseiller le ministre de l'Éducation sur toute question relative à l'éducation, s'est spécifiquement intéressé au moins à deux occasions aux SGMS (CSÉ, 2006 et 2012). Des défis à relever ont été proposés pour améliorer la qualité des SGMS et des leviers ont été retenus pour y arriver. Rehausser les compétences du personnel des SGMS fait partie de l'un des six leviers que proposait en 2006 le Conseil supérieur de l'éducation pour améliorer la qualité des services offerts : "Rehausser les exigences de formation pour l'obtention d'un poste et favoriser l'accès à la formation continue. » (CSÉ, 2006, p. 61). « Le Conseil estime que la mission des services de garde en milieu scolaire ne peut se réaliser pleinement qu'à la condition que le personnel possède les compétences nécessaires pour la mettre en œuvre » (CSÉ, 2006, p. 61). Parmi les compétences essentielles pour le personnel des SGMS, nous retrouvons la compétence à communiquer oralement (Berger et al., 2012; Berthiaume, 2016). Les rares recherches qui se sont intéressées à cette compétence mentionnent la nécessité de développer cette dernière chez le personnel des SGMS (Myre-Bisaillon \& Chalifoux, 2013).

Actuellement, des recherches sont nécessaires afin de documenter les compétences et le rôle éducatif des éducateurs en SGMS (Manningham, 2008; Chalifoux et al., 2014). Plus précisément, on parle d'un vide théorique en ce qui concerne la compétence à communiquer oralement du personnel des SGMS (Bergeron et al., 2015) et d'une priorité dans les recherches à venir (Myre-Bisaillon \& Chalifoux, 2013). La qualité des interventions à l'oral avec les enfants et avec les parents est d'une très grande importance (CSÉ, 2006; Berger et al., 2012) et nécessite qu'on s’y attarde afin de pouvoir mieux encadrer et former le personnel des SGMS (CSÉ, 2006; Manningham, 2008). Ce constat nous a amenés à nous poser la question de recherche suivante : quelles sont les perceptions du personnel d'un SGMS d'une école primaire montréalaise quant à sa compétence à communiquer oralement?

\section{Cadre théorique}

Pour pouvoir bien fonctionner dans la société et s’adapter aux nombreux changements qui peuvent survenir sur le marché du travail, tout individu doit avoir un niveau de compétences en littératie suffisamment élevé et être en mesure de poursuivre le développement de ces dernières dans une perspective d'apprentissage tout au long de la vie (Dumais, 2015; Lafontaine \& Pharand, 2015). Nous entendons par littératie « la capacité d'une personne, d'un milieu et d'une communauté à comprendre et à communiquer de l'information par le langage sur différents supports pour participer activement à la société dans différents contextes » (Lacelle, Lafontaine, Moreau \& Laroui, 2016, p. 1). Parmi les compétences qui font partie du concept de littératie, la compétence à communiquer oralement est reconnue comme une composante essentielle de ce concept (Allen, 2014; Soucy, 2016). En effet, savoir communiquer oralement est une compétence de premier plan dans la société actuelle (Dumais, 2015). Elle « englobe les contextes de prise de parole, la production orale, le vocabulaire compris et utilisé, la compréhension d'un message, la capacité d'écoute, les interactions sociales dans des situations de communication scolaires ou extrascolaires ainsi que les liens étroits entre ces paramètres » (Lafontaine \& Pharand, 
2015, p. 3), des éléments essentiels à maitriser pour le personnel des SGMS (Berthiaume, 2016; Soucy \& Dumais, 2017).

Tous les acteurs de l'école doivent avoir une maitrise suffisante de la compétence à communiquer oralement, dont le personnel des SGMS (Berger et al., 2012). En effet, ce personnel joue un rôle de modèle linguistique auprès des enfants. " Il est donc important que les différents intervenants qui interagissent auprès de l'enfant s'attardent à la qualité de leur propre langage » (Cloutier, 2012, p. 75). Ce rôle de modèle linguistique est encore plus important en milieu plurilingue et en milieu défavorisé puisque l'école peut être le seul endroit où le français est utilisé et où un registre de langue standard peut être entendu (CSÉ, 1996). De plus, certains enfants peuvent passer autant de temps en classe qu'au SGMS dans une journée (Myre-Bisaillon, Chalifoux, Lapointe-Garant, Dionne \& Rodrigue, 2014; Dumais \& Soucy, 2016). La compétence à communiquer oralement du personnel des SGMS peut donc avoir une influence considérable sur cette même compétence chez les enfants. Également, puisque le SGMS a le mandat de poursuivre le projet éducatif de chaque école où il est implanté, le personnel des SGMS est considéré comme un partenaire du personnel enseignant dans le développement global de l'enfant (CSÉ, 2006; Myre-Bisaillon et al., 2014). Ce partenariat permet également le développement des compétences transversales au cœur de l'école québécoise, ce qui inclut la compétence reliée à la communication (Ministère de l'Éducation du Québec, 2001; Berger et al., 2012).

Le personnel des SGMS joue aussi un rôle important dans la relation école-famillecommunauté, notamment en raison des interactions orales avec les parents (CSÉ, 2006; Berger et al., 2012). De façon générale, les éducatrices et les éducateurs des SGMS « ont la possibilité de parler aux parents matin et soir, d'en connaitre un peu plus sur la famille, sur les habitudes des enfants et sur les méthodes d'intervention choisies à la maison » (Berger et al., 2012, p. 50). Ces contacts avec les parents s'avèrent encore plus déterminants en milieu plurilingue et pluriethnique (CSÉ, 2006; Berger et al., 2012). En effet, pour plusieurs familles immigrantes, le personnel des SGMS est le premier contact avec la culture québécoise et le contact le plus régulier, faisant en sorte que ce personnel devient une référence et contribue, de façon volontaire ou non, à l'intégration de ces familles au système scolaire québécois et à la société québécoise (Conseil de la famille et de l'enfance, 2006). Le personnel des SGMS est donc un acteur de premier plan en ce qui concerne le lien entre l'école et la famille (le personnel fournit diverses informations sur les enfants et l'école aux parents), et cela passe par la communication orale (Baillargeon, Caouette \& Darveau, 1990).

Puisque la compétence à communiquer oralement est une compétence importante dans le travail du personnel des SGMS (rôle de modèle linguistique, interaction avec les enfants et les parents, etc.) et que nous en savons très peu à propos de cette compétence spécifique chez ce personnel scolaire, nous avons souhaité explorer cette zone d'ombre de la recherche. Nous avons donc mené une recherche exploratoire qui avait comme objectif de décrire les perceptions du personnel d'un SGMS d'une école primaire montréalaise quant à sa compétence à communiquer oralement. Cette exploration des perceptions de cette compétence apportera un nouvel éclairage, c'est-à-dire des données scientifiques, qui pourront être un point de départ pour améliorer la formation initiale et continue du personnel des SGMS et, par le fait même, les compétences en littératie de ce personnel. 


\section{Méthodologie}

Cette section présente la méthodologie employée pour répondre à l’objectif de recherche, soit décrire les perceptions du personnel d'un SGMS d'une école primaire montréalaise quant à sa compétence à communiquer oralement.

Type de recherche

Nous avons mené une recherche de type qualitatif/interprétatif (Savoie-Zajc, 2011) et de nature descriptive (Gaudreau, 2011). Elle peut également être qualifiée d'exploratoire (Savoie-Zajc, 2011). En effet, la recherche exploratoire est utilisée par les chercheurs lorsqu'ils possèdent très peu d'informations préalables sur une situation ou un objet de recherche et qu'ils souhaitent en avoir une compréhension préliminaire, ce qui est notre cas.

\section{Sélection du SGMS}

Pour mener cette recherche exploratoire, nous souhaitions travailler avec un SGMS qui avait plus de 10 personnes à son emploi pour nous garantir un minimum de participants. Une invitation a été lancée par courriel à l'automne 2015 à toutes les directions d'écoles primaires d'une commission scolaire de l'ile de Montréal par une personne responsable de la recherche dans cette commission scolaire. Nous avons obtenu une seule réponse à cette invitation. C'est donc ce SGMS qui a été choisi. Il s'agit d'un échantillonnage de convenance (Gaudreau, 2011; Fortin \& Gagnon, 2016).

\section{Sujets de la recherche}

Cette recherche a été menée à l'hiver 2016 auprès de 12 employés d'un SGMS d'une école primaire en milieu défavorisé, plurilingue et pluriethnique de l'ile de Montréal. Parmi les 12 sujets, nous retrouvons 1 technicien (responsable du service de garde), 6 éducateurs en service de garde ( 5 femmes et 1 homme), 4 surveillants du diner ( 3 femmes et 1 homme) ainsi que 1 femme qui occupe un emploi d'éducatrice en SGMS en fin de journée dans l'école où a eu lieu la recherche et un emploi de surveillante du diner dans une autre école. Les 12 sujets ont des formations variées. Le tableau 1 présente l'ensemble des formations déclarées selon l'emploi occupé pour les 12 sujets. 
Tableau 1

Formations déclarées selon l'emploi occupé pour les 12 sujets

\begin{tabular}{|c|c|}
\hline Emploi & Formation \\
\hline $\begin{array}{l}\text { Responsable du } \\
\text { service de garde, } \\
\text { éducatrice et } \\
\text { éducateur en } \\
\text { service de garde }\end{array}$ & $\begin{array}{l}\text {-Attestation d'études professionnelles (AEP) : } \\
\text { service de garde (2) } \\
\text {-Diplôme d'études professionnelles (DEP) : } \\
\text { cuisine (1); pâtisserie (1); service de garde (2) } \\
\text {-Attestation d'études collégiales (AÉC) : } \\
\text { éducation à l'enfance (2) } \\
\text {-Diplôme d'études collégiales (DEC) : } \\
\text { sciences humaines (1); interventions en loisir (1); } \\
\text { service de garde (1) } \\
\text {-Certificat : } \\
\text { petite enfance (1); préposée aux bénéficiaires (1); } \\
\text { bureautique (1) } \\
\text {-Baccalauréat : } \\
\text { éducation préscolaire et enseignement primaire (1); } \\
\text { enseignement de l'espagnol (1) }\end{array}$ \\
\hline $\begin{array}{l}\text { Surveillante et } \\
\text { surveillant du } \\
\text { diner }\end{array}$ & $\begin{array}{l}\text {-Attestation d'études collégiales (AEC) : } \\
\text { service de garde (1) } \\
\text {-Diplôme d'études collégiales (DEC) : } \\
\quad \text { écologie (1) } \\
\text {-Baccalauréat : } \\
\quad \text { psychologie (1); gestion et commerce (1) }\end{array}$ \\
\hline
\end{tabular}

Les 12 sujets ont indiqué avoir une ou plusieurs de ces langues comme langue maternelle : français (4), arabe (2), berbère (2), anglais (1), créole (1), espagnol (1), portugais (1), tamoul (1) et iranien (1). Ils ont en moyenne 5,1 années d'expérience de travail dans un SGMS. Les éducatrices et éducateurs en service de garde travaillent en moyenne 23 heures par semaine alors que les surveillantes et surveillants du diner travaillent en moyenne 7,75 heures par semaine. Sept sujets ont mentionné n’avoir jamais suivi de formation sur la langue orale ou la compétence à communiquer oralement, quatre ont indiqué avoir suivi une formation sur la langue orale ou la compétence à communiquer oralement et un sujet n’a pas répondu à cette question.

Collecte des données

La collecte des données a été effectuée à l'aide d'un questionnaire, d’observations non participantes et d'entrevues semi-dirigées.

\footnotetext{
${ }^{3}$ Nous avons inclus le technicien (responsable du service de garde) aux éducatrices et éducateurs en service de garde pour éviter que ce sujet puisse être identifié. De plus, nous avons inclus l'éducatrice en service de garde qui occupe aussi un emploi de surveillante du diner dans une autre école à cette catégorie pour éviter qu'elle puisse être identifiée et parce qu'elle occupait un emploi d'éducatrice en service de garde dans l'école où a eu lieu la recherche.
} 
Le questionnaire comportait trois sections. Une première section collectait des données sociologiques (sexe, formation, type d'emploi, années d'expérience, langue maternelle, etc.). La deuxième section était constituée de quatre questions qui comportaient chacune plusieurs affirmations où les sujets devaient déterminer une réponse à l'aide d'une échelle de Likert à quatre choix ( «très à l'aise ", «à l'aise ", " plus ou moins à l'aise ", « pas du tout à l'aise »), et ce, afin d'éviter un biais de tendance centrale. Ils avaient la possibilité de répondre "N/A » lorsque l'affirmation ne s'appliquait pas à eux. Cette deuxième section comportait aussi trois questions à développement (les sujets devaient expliquer leurs réponses à l'écrit). Les questions de cette deuxième section du questionnaire ont été inspirées en partie de questionnaires utilisés dans le cadre de recherches sur la compétence à communiquer oralement de futurs enseignants (Mottet, 2009; Messier, Dumais \& Viola, 2012) et d'écrits sur le travail effectué par le personnel des SGMS (Conseil de la famille et de l'enfance, 2006; Sorel Étienne et al., 2015). Enfin, la troisième section demandait aux sujets s'ils souhaitaient participer à la phase deux de la recherche. Elle consistait à faire une entrevue semi-dirigée et à enregistrer leurs interactions orales, lors de leur quart de travail, pendant deux journées, à l'aide d'un micro-cravate et en présence d'un chercheur. Sur une possibilité de 17 membres du personnel du SGMS (8 éducatrices et éducateurs, 1 technicien et 8 surveillantes et surveillants du diner), 12 personnes ont accepté ${ }^{4}$ de répondre au questionnaire. Mentionnons que le questionnaire a été validé auprès de trois éducatrices en SGMS d'une autre commission scolaire avant d'être utilisé dans le cadre de cette recherche. Les éducatrices qui ont participé à la validation ont mentionné la nécessité de bien contextualiser la recherche et de faire part des intentions de cette dernière pour éviter que le personnel du SGMS se sente jugé. Nous avons tenu compte de ces remarques lors de la passation du questionnaire. Nous avons également revu la présentation de certaines questions et expliqué quelques mots qui n’étaient pas clairs pour elles.

Une observation non participante a été effectuée. Elle consistait à observer, à enregistrer à l'aide d'un micro-cravate et d'un enregistreur portatif, et à noter dans une grille d'observation ce que les chercheurs voyaient et entendaient sans intervenir dans les activités des participants et en se tenant à l'écart. L'observation avait pour but de décrire « les comportements et les expériences des personnes comme ils se présentent dans leurs milieux naturels » (Fortin \& Gagnon, 2016, p. 201). Les deux chercheurs souhaitaient donc mieux comprendre le travail du personnel de ce SGMS et les réponses fournies dans le questionnaire en plus de pouvoir observer en temps réel la compétence à communiquer oralement des sujets. Ils ont noté tant ce qu'ils entendaient que ce qu'ils voyaient (ce qui inclut les éléments non verbaux de l'oral comme les gestes et le regard). Quatre sujets ont accepté d’être observés et enregistrés : une éducatrice en service de garde, un technicien ainsi qu'une surveillante du diner et un surveillant du diner. Les observations ont eu lieu pendant huit jours, à raison de deux journées d'observation par sujet. Chacun des chercheurs observait un sujet à l'une des deux journées. Les chercheurs étaient présents

\footnotetext{
${ }^{4}$ Malgré toutes les mesures mises en place pour expliquer la recherche et pour rassurer les participants quant à la confidentialité des données, cinq personnes ont refusé de répondre au questionnaire par peur de perdre leur emploi (propos émis par les personnes). En effet, quatre personnes sur cinq étaient surveillantes et surveillants du diner, un emploi précaire, et les cinq personnes n’avaient pas le français comme langue première. Elles avaient peur d'être jugées en ce qui concerne leur compétence à communiquer à l'écrit et à l'oral, et que cela ait une incidence sur leur emploi.
} 
pendant tout le quart de travail des sujets (de $7 \mathrm{~h}$ à $18 \mathrm{~h}$ pour certains et 90 minutes seulement pour d'autres). Mentionnons que les deux chercheurs se sont exercés à observer dans une autre école pendant une demi-journée avant d'observer les quatre participants. Cela a permis de valider la grille d'observation et de comparer les observations écrites des deux chercheurs afin d'assurer une certaine uniformité entre celles-ci tant en ce qui concernait le contenu que la façon de noter.

Après avoir fait l'analyse des données du questionnaire et après l'observation des quatre sujets, les chercheurs ont mené des entrevues. Il s'agissait d'entrevues semi-dirigées à l'aide de questions ouvertes dans le but de mieux comprendre les réponses du questionnaire ainsi que les observations effectuées chez les quatre sujets observés. Parmi les 12 sujets qui ont répondu au questionnaire, 8 ont accepté de faire des entrevues (1 technicien, 4 éducatrices et éducateurs en service de garde, ainsi que 3 surveillantes et surveillants du diner), ce qui inclut les 4 sujets observés.

\section{Analyse des données}

Pour ce qui est de l'analyse des données, c'est principalement l'analyse de contenu qui a été utilisée (L’Écuyer, 1987; Bardin, 2009). Des unités de sens ont été sélectionnées à partir des réponses des questions à développement du questionnaire, des verbatim des entrevues et des grilles d'observation à la suite des observations non participantes, et ce, afin de leur attribuer un ou plusieurs descripteurs. Pour le choix des descripteurs, le modèle mixte a été privilégié, car il permet d'avoir des descripteurs préexistants auxquels d'autres sont ajoutés en cours d'analyse ou sont retirés (L’Écuyer, 1987; Sauvé, 1992). Les descripteurs préexistants ont été déterminés à partir des thèmes des questions du questionnaire. Pour nous assurer de la fiabilité interne du codage, nous avons procédé à un recodage intrajuge (Miles \& Huberman, 2003). Quant aux réponses chiffrées du questionnaire (selon une échelle de Likert), nous avons utilisé le logiciel Excel pour compiler les données.

\section{Résultats}

Dans cette section, les résultats de la recherche sont présentés selon sept grands thèmes qui ont émergé de l'analyse de contenu. Les données issues du questionnaire, des observations et des entrevues semi-dirigées sont donc exposées conjointement.

\section{La responsabilité du personnel du SGMS quant à la langue orale des élèves}

Dans le questionnaire, lorsqu'on demande aux éducateurs et aux surveillants ${ }^{5}$ s'ils croient avoir une responsabilité en ce qui concerne le développement de la langue orale des élèves, les 12 répondants affirment que oui. L'explication la plus commune semble être que ces derniers considèrent qu'ils ont un rôle éducatif à jouer. À cet égard, un participant rapporte même qu'il porte une certaine responsabilité, car cela fait partie du développement global des élèves. Lorsqu’on s’attarde au contenu des entrevues, les réponses données par le personnel du service de garde viennent confirmer les réponses du questionnaire : « Je pense que quand tu passes 4-5 heures par jour avec un enfant [...], je pense que oui, pour

\footnotetext{
${ }^{5}$ Nous utiliserons maintenant seulement le masculin lorsqu'il est question d'éducateurs et d'éducatrices ainsi que de surveillants et de surveillantes du diner afin d'alléger le texte et d'assurer la confidentialité des sujets.
} 
moi je fais comme si j’étais un prof, c'est important de bien s'exprimer avec les enfants ${ }^{6}$. » $\left(\mathrm{S}^{7}\right)$ et « Évidemment parce que nous sommes une complémentarité de l'éducation de ces élèves qui nous sont confiés après bien sûr l'autorité parentale et les enseignants » (E1).

\section{Être un modèle linguistique}

Dans le questionnaire, nous avons demandé aux répondants s’ils pensaient que leur propre façon de s'exprimer à l'oral avait une influence sur la langue orale qu'utilisent les enfants. Neuf sujets ont répondu par l'affirmative alors que trois sujets (un surveillant du diner et deux éducateurs) ne pensaient pas avoir d'influence sur la langue orale des enfants. Toutefois, seulement huit personnes ont donné une réponse explicative et il s'agit de répondants qui croyaient avoir une influence. Certains considèrent que leur influence vient du fait qu'ils sont des modèles pour les élèves : « Lorsque je m'adresse aux enfants, je suis un modèle, alors j'ai beaucoup d'influence » (E3) et du fait que les enfants ont tendance à répéter ce qu'ils disent : «Si on leur donne une consigne, les enfants répètent souvent mots pour mots ce qu'on leur a dit » (S2). D'autres font ressortir qu'ils peuvent guider et corriger les élèves : "Oui, car je peux corriger ou guider leurs choix de mots pour s'exprimer » (S4).

Les résultats de l'entrevue abondent dans le même sens. À l'exception d'un surveillant du diner, l'ensemble du personnel interrogé considère qu'il est important d'avoir un langage adéquat afin d'être un modèle auprès des enfants. Le personnel reconnait que les enfants auront tendance à reproduire ce qu'ils entendent, comme l'explique S2 : «... ils absorbent qu'est-ce qu'on dit, de la manière qu'on le dit, ils vont le dire tel quel après, si on le dit tout croche, ils vont nous le dire tout croche ». Puisque les enfants de l'école où a eu lieu la recherche ont rarement le français comme langue maternelle, il est essentiel d'utiliser un vocabulaire précis et d'émettre des messages clairs selon les sujets qui ont participé aux entrevues. Parmi les gens interrogés, seul un surveillant (S3) du diner ne semble pas se préoccuper d'être un modèle linguistique. Selon ses propos, le respect que les élèves ont à son égard est plus important que le modèle qu'il pourrait être dans sa façon de communiquer et dans le choix de ses mots. Considérant le peu de temps qu'il passe avec les enfants et surtout le peu de discussions qu'il entretient avec eux, il ne croit pas que sa manière de s'exprimer puisse avoir une influence sur la communication orale des élèves.

«Les enfants respectent beaucoup leur surveillant de diner. Est-ce qui prend le surveillant de diner comme un adulte qui veut le suivre l'exemple? Moi je dis que non, la plupart non. C'est juste un surveillant de diner, c'est juste quelqu'un qui est là pendant l'heure du lunch, et puis qui regarde, et puis qui chicane quand il fait quelque chose qui n'est pas bon. Alors non, pas beaucoup d'influence, mais ça dépend aussi du surveillant »(S3).

\footnotetext{
${ }^{6}$ Nous retranscrivons chaque fois les propos tels qu’ils ont été écrits dans les questionnaires et entendus dans les entrevues.

${ }^{7}$ Lorsque des verbatims ou des extraits du questionnaire sont présentés, nous utilisons la lettre « $\mathrm{S}$ » accompagnée d'un chiffre pour indiquer qu'il s'agit de propos de surveillantes ou des surveillants du diner et la lettre « E » accompagnée d'un chiffre pour indiquer qu'il s'agit d'une éducatrice ou d'un éducateur en service de garde ou bien du technicien.
} 
Un éducateur (E3) mentionne qu'il serait pertinent d'utiliser la langue française en tout temps à l'école, car il constate parfois que certains membres du personnel du SGMS se parlent entre eux dans une autre langue que le français, ce qui, selon ses dires, n'offre pas un bon modèle et n'encourage pas l'apprentissage du français pour les enfants : « [les] éducatrices ou éducateurs, dans la cour d'école, parlaient souvent entre eux leur langue, alors ça favorise pas, je trouve, les, en tout cas les, la communication en français oral. » (E3) Cela pourrait aussi occasionner des difficultés dans la résolution de conflits pour les enfants.

« En principe on va s’adresser en français lorsqu'il y a un problème tout ça, mais ce qui arrive c'est que souvent, c'est que admettons les enfants qui sont russes s’il y a une éducatrice russe va parler à l'enfant qu'il y a une petite bagarre, quelque chose, elle va sûrement parler en russe. Et des fois, l'autre enfant, il n’est pas russe alors il ne comprend même pas ce qui se passe, alors ça coupe. Moi je trouve, ça coupe la communication aussi la résolution de conflits » (E3).

Lors des observations, nous avons entendu à quelques reprises des membres du personnel s'exprimer dans une autre langue que le français en présence des enfants. De plus, nous avons pu observer que certains membres du personnel portaient une attention particulière à la langue orale. Trois des quatre personnes observées ont fait des interventions au niveau de la langue orale des enfants : reformulation de leurs propos pour être mieux compris et demande de reformulation aux enfants pour mieux comprendre, demande de précisions, utilisation du mot juste, recherche d'un mot en français pour remplacer l'utilisation d'un mot en anglais, échanges sur la meilleure façon de dire une insatisfaction à un ami et aux parents, etc. Par exemple, un sujet a demandé à un enfant de préciser ce qu'il souhaitait dire puisqu'il utilisait le déictique "ça » et le mot " affaire » pour parler d'un vêtement d'hiver (tuque) et qu'il était difficile de comprendre le propos. À un autre moment, un des participants observés a reformulé les paroles d'un enfant pour s'assurer de sa compréhension. L'enfant a dû redire en d'autres mots son propos puisque la reformulation du participant ne reflétait pas ce que l'enfant souhaitait exprimer. L'enfant a donc utilisé d'autres mots pour mieux se faire comprendre.

\section{La capacité à s'exprimer clairement et correctement à l'oral}

Dans le questionnaire, nous avons posé la question suivante aux 12 sujets : «De façon générale, vous considérez-vous comme une personne qui s’exprime clairement et correctement à l'oral? Expliquez. » Les 12 sujets ont répondu par l'affirmative, mais seulement 6 personnes ont expliqué par écrit pourquoi. Dans leurs explications, les sujets semblent associer « une expression claire et correcte à l'oral » à la compréhension qu'ont les enfants de leurs consignes : "Parce que les enfants écoutent très bien mes consignes par la suite. » (E6) et «J'aissaye [sic] de donner des consignes claires avec des mots adaptés à l'âge des enfants » (E3). Pour un surveillant du diner, une expression claire et correcte à l'oral semble essentiellement être associée à la compréhension du propos par les enfants : "Mon français est accente et occaisionellement gramaticament pas correct mais je parle dans un façon que les enfants me comprendre » (S3).

Les entrevues ont permis d'en apprendre davantage à ce propos. Entre autres, le surveillant du diner S3 a nuancé sa réponse en faisant des liens avec les apprentissages que les enfants pourraient faire en l'entendant parler : «Je peux pas dire que les enfants vont 
apprendre à parler français correctement à cause qu'ils suivent mon exemple. Je peux le donner un exemple dans un autre sens, mais pas parler français correct. C'est pas mon capacité de faire ça, parce que je ne suis pas assez bon en français pour faire ça » (S3). Un autre surveillant est conscient qu'il utilise des mots en anglais, mais cela ne change pas sa perception quant à sa capacité de s'exprimer clairement et correctement à l'oral : « Je pense que je parle quand même assez correctement. Je fais pas trop d'erreurs, des fois c'est plus facile des mots en anglais, surtout "le fun", ou des anglicismes qu'on est habitué de dire, mais habituellement j'essaye de parler bien avec les enfants » (S6).

Lors des observations que nous avons menées et lors des entrevues, nous avons parfois eu de la difficulté à bien comprendre les propos du personnel du SGMS et certains faisaient des erreurs à l'oral (entre autres en ce qui a trait aux accords en genre et en nombre et au choix des mots) selon ce qui pourrait être attendu d'un modèle linguistique (Mottet \& Gervais, 2007).

Les personnes qui influencent la communication orale des enfants

Dans le questionnaire, nous avons demandé aux 12 répondants d'indiquer le niveau d'influence de 6 personnes sur la communication orale des enfants. Ils devaient indiquer sur une échelle de 1 à 4 le niveau d'influence de ces personnes, 1 étant beaucoup d'influence et 4 peu d'influence. Nous avons calculé le pointage obtenu pour chacune des personnes (plus le pointage est bas, plus la personne a de l'influence sur la communication orale des enfants et plus le pointage est élevé, moins elle a d'influence). Les enseignants (12 points) et les parents (13 points) seraient les personnes ayant le plus d'influence sur la communication orale des enfants selon les 12 répondants. Les autres enfants suivraient de près avec 17 points. Ce serait les surveillants du diner (27 points) qui auraient le moins d'influence sur la communication orale des enfants selon les 12 répondants. La direction d'école (24 points) arriverait en cinquième position, précédée par les éducateurs en quatrième position (23 points) (voir tableau 2).

Tableau 2

Ordonnancement des personnes qui ont le plus d'influence sur la communication orale des enfants

\begin{tabular}{|c|l|}
\hline Position & \multicolumn{1}{|c|}{ Personnes } \\
\hline 1 & Les enseignants \\
\hline 2 & Les parents \\
\hline 3 & Les autres enfants \\
\hline 4 & Les éducateurs \\
\hline 5 & La direction de l'école \\
\hline 6 & Les surveillants du diner \\
\hline
\end{tabular}

Nous avons questionné les huit sujets qui ont accepté de participer aux entrevues afin de mieux comprendre ce classement. À la suite de l'analyse des entrevues, ces résultats pourraient, entre autres, s'expliquer par le temps passé par les personnes en compagnie des enfants. En effet, quatre des participants de l'entrevue ont clairement mentionné que ceux qui passent le plus de temps avec les enfants ont plus d'influence sur leur communication orale : «Moi, je pense qu'ils [les parents] ont une influence là, c'est sûr que naturellement 
ils passent plus de temps les enfants avec leurs parents » (E2). La même explication est donnée par E5 pour expliquer le fait que les éducateurs ont une plus grande influence que les surveillants du diner : " je pense que les éducateurs c'est beaucoup plus, parce que je fais un poste de surveillante de diner dans une autre école, éducatrice ici, je vois que les enfants ici, ils m'écoutent mieux parce que je passe beaucoup plus de temps avec eux, parce que le diner, je passe à peu près une heure ". Un surveillant abonde dans le même sens : " Les éducateurs ils ont beaucoup plus de temps avec les enfants qu'un surveillant » (S3). Pour E2, les membres de la direction de l'école ont moins d'influence, car les enfants « vont juste leur avoir dit bonjour une fois dans l'année puis c’est tout » et « y’ont pas à intervenir à tous les jours avec eux " (E2). Une autre explication de ce classement concernerait le statut de la personne dans l'école. Les enseignants auraient une plus grande influence sur la communication orale des élèves de par la valeur que ces derniers accordent à leur enseignant : "... ils sont beaucoup plus attentifs aux enseignants par rapport aux éducatrices, aux surveillants du diner... » (E5).

\section{Le niveau d'aisance à communiquer dans différentes situations}

Dans le questionnaire, trois questions concernaient le niveau d'aisance à communiquer oralement dans des contextes 1) impliquant des enfants, 2) impliquant des parents et 3) impliquant des collègues. Une liste de situations était associée à chacun des contextes et les sujets devaient indiquer de 1 à 4 leur niveau d'aisance (1: très à l'aise; 2 : à l'aise; 3 : plus ou moins à l'aise; 4 : pas du tout à l'aise; N/A : ne s'applique pas). Les résultats du questionnaire nous indiquent que le personnel est davantage à l'aise de communiquer avec les enfants. C'est la communication avec les autres collègues qui leur semble la moins aisée. Voici les contextes en ordre d'aisance selon les huit sujets et quelques explications.

1. Aisance à communiquer dans des contextes impliquant les enfants.

Ils sont à l'aise ou très à l'aise dans presque toutes les situations. Ils sont un peu moins à l'aise de communiquer lorsqu'il faut gérer les déplacements dans l'école et lorsqu'il y a un conflit entre eux et l'enfant.

2. Aisance à communiquer dans des contextes impliquant les parents.

Ils sont à l'aise ou très à l'aise d'entrer en contact avec les parents ou de leur parler positivement de leur enfant. Ils sont cependant plus ou moins à l'aise lorsqu'il faut rapporter des évènements négatifs.

3. Aisance à communiquer dans des contextes impliquant des collègues.

Ils sont plus ou moins à l'aise lorsqu'ils doivent régler un problème entre eux et les enseignants. Ils sont encore moins à l'aise lorsqu'il y a un conflit entre eux et un autre éducateur ou surveillant. Mentionnons qu'ils sont plus ou moins à l'aise de prendre la parole lors des rencontres de travail.

Les entrevues nous ont permis de mieux comprendre cet ordonnancement. En ce qui concerne les situations avec les enfants, malgré quelques moments propres à chaque sujet où la communication orale peut être plus difficile, le personnel trouve habituellement des façons efficaces de communiquer avec les enfants selon leurs dires. Pour ce qui est de la communication avec les parents, elle serait majoritairement positive étant donné la bonne 
entente entre le personnel du SGMS et les parents. Ce qui compliquerait la communication et rendrait mal à l'aise le personnel, c'est lorsqu'il faut communiquer des éléments négatifs aux parents, lorsqu'il faut réclamer de l'argent et lorsque la communication doit se faire dans une autre langue que le français. Un éducateur (E2) mentionne que :

« La problématique c’est plus au niveau de la langue. Il y a beaucoup de parents qui parlent pas français, donc c’est plus là qui est problématique. Tu veux pas non plus les énerver, les affoler, donc faut choisir les bons mots, mais est-ce que le bon mot pour toi est-ce qu'ils le comprennent pour eux dans notre langue, versus dans leur langue. C’est pas toujours évident. »

Pour ce qui est du niveau d'aisance à communiquer oralement avec les collègues, les entrevues ont permis de mieux comprendre ce qui posait problème avec les collègues du SGMS, mais aussi avec les enseignants. Avec les collègues du SGMS, les sujets ont principalement mentionné qu'il était souvent difficile de parler d'un problème avec un collègue, car ils ont peur d'être en conflits par la suite avec la personne :

« Je veux pas en parler parce que je veux pas avoir de problèmes directement avec la personne, je vais voir mon supérieur puis je vais voir la direction, si jamais c'est quelque chose de grave, je vais peut-être dire une phrase ou deux, à la personne concernée, mais je vais pas toute lui dire, je vais juste dire le strict minimum [...] Faut que tu fasses attention à ce que tu leur dis parce que s’ils sont fâchés avec toi, après ça, ils vont pas t'aider, c'est plus complexe, puis je préfère me taire que de parler avec un collègue, donc je me tais » (S2).

En ce qui concerne l'aisance à communiquer avec les enseignants, cela varie beaucoup d'un sujet à un autre. Certains affirment ne pas avoir de contacts avec les enseignants alors que d'autres ont l'occasion à certains moments de communiquer avec eux. Le problème se situerait au niveau de la fréquence des communications avec les enseignants. Les sujets ont indiqué ne pas avoir de temps prévu à l'horaire pour communiquer avec eux. Cela aurait pour conséquence que le personnel du SGMS ne serait pas mis au courant d'éléments qu'il juge importants :

« On est même pas mis au courant, là des fois tu te dis, avoir su, tel enfant je n’aurais pas agi de cette façon-là, nos interventions seraient meilleures avoir l’info. On n’a pas besoin de savoir tout, mais au moins avoir l'essentiel. Dire regarde, il y a eu telle situation familiale, il parle pas de son père là parce que son père a battu sa mère, puis là il est en prison » (E2).

Un éducateur a mentionné avoir appris seulement deux mois après la rentrée scolaire qu’un élève avait un trouble du spectre de l'autisme, alors que cela aurait été connu de l'école depuis le début de l'année. Les sujets mentionnent que les rares occasions où ils sont en présence des enseignants, ce ne sont pas de bons moments pour parler des élèves, ce qui n'aide pas à échanger avec les enseignants : «Le seul temps qu'il y a c'est au début de l'année quand on fait le diner là on a aucun enfant, puis le reste du temps on a toujours [les enfants]. Le party de Noël, c'est pas le temps de parler d'élèves, c'est pas le bon moment si on peut dire » $(\mathrm{S} 2)$. 


\section{La collaboration entre le personnel du SGMS et les enseignants}

Dans le questionnaire, aucune question n'abordait spécifiquement la collaboration entre le personnel des SGMS et les enseignants. Toutefois, à la suite de nos observations, nous avons souhaité questionner les participants à ce sujet lors des entrevues. En effet, pendant nos journées d'observations, aucun échange n’a eu lieu entre les enseignants et le personnel du service de garde. Lorsque les enseignants sont venus chercher les élèves à l'extérieur, au son de la cloche, ni les surveillants ni les éducateurs n'ont communiqué avec les enseignants. De plus, à la fin de la journée, les enseignants ne se sont pas déplacés vers le service de garde pour communiquer certaines informations aux éducateurs. Le seul échange qu'il nous a été possible d'observer fut un enseignant qui a ramené trois fillettes en disant que la mère n'était pas venue les chercher. Sans plus d'explications, l'enseignant est reparti, laissant à l'éducateur la charge de trois fillettes supplémentaires, sans que ces dernières soient inscrites dans son groupe. Il a donc dû faire appel au responsable du service de garde. À un autre moment, lors des observations, nous avons été témoins d'une bataille entre élèves à l'heure du diner où un surveillant du diner est intervenu. Nous avons questionné ce dernier pour savoir ce qu'il avait fait comme suivi auprès des enseignants. Il nous a indiqué qu'aucun suivi n'avait été fait puisqu'aucun moment n'était prévu pour parler aux enseignants.

Lors des entrevues, nous avons demandé aux huit participants s'ils croyaient que la collaboration avec les enseignants était importante. Ils ont été unanimes à ce sujet : la collaboration avec les enseignants est primordiale. Les deux principales raisons évoquées sont le suivi et la cohérence. Les sujets croient qu'il est nécessaire de bien collaborer, car le suivi auprès des enfants s'en voit facilité. $\mathrm{S} 1$ : « ... par exemple, au diner, on communiquait au niveau si un enfant n'allait pas bien une matinée, [l'enseignante] me tenait au courant, me disait aujourd'hui tel ou tel je crois qu'il n'a pas bien dormi, je pense que ça va pas très bien aujourd'hui, c'est très très important, parce que ça facilite la tâche pour les deux, et pour l'enfant aussi c'est plus agréable. » Selon les sujets, la collaboration est importante, car elle peut permettre une certaine cohérence au niveau des règles. S3 explique qu'il est important « ... qu’on donne pas des conseils différents à les enfants. Les enseignants dit non, le règlement sont comme ça, et si l'éducateur décide non, le règlement va être complètement différent, c'est dur d'avoir, un va empêcher le respect... » Lors des entrevues, nous avons aussi demandé ce qui nuisait à la collaboration. La réponse la plus entendue fut le manque de temps. Les éducateurs et les surveillants s'entendent pour dire qu'il y a peu de temps prévu pour faire des suivis ou pour discuter avec les enseignants, ce qui nuirait à la collaboration.

\section{Les besoins de formation}

À la suite du questionnaire et des observations, nous avons souhaité savoir si les huit sujets qui participaient aux entrevues considéraient importante la formation continue. Ils ont tous répondu que la formation était importante pour eux : « Pour moi la formation c'est important, qui dit formation pour moi dit important » (E1). Certains ont cependant mentionné qu'il était difficile d'avoir accès à des formations en raison du budget, du manque de personnel pour remplacer ceux qui vont en formation et en raison de l'ouverture de la direction dans certains milieux. Un surveillant du diner mentionne que «ça dépend toujours de la direction. Moi, je me rappelle à d'autres places, j'ai juste voulu aller suivre une formation, puis la directrice, elle voulait pas parce que c'est pas dans ta tâche, tu t'en serviras pas nécessairement » (S2). 
Lors des entrevues, nous avons demandé aux sujets de nous indiquer, selon eux, l’ordre de priorité des éléments à aborder dans le cadre d'une formation sur les interactions. Parmi cinq choix, voici l'ordre de priorité des huit sujets : 1. Les interactions avec les enfants; 2. Les interactions entre collègues; 3 . Les interactions avec les enseignants; 4 . Les interactions avec les parents; 5. Les interactions avec la direction. Les interactions avec les enfants sont prioritaires, car selon E4 «Ça c'est notre job. Nous sommes ici pour les enfants, il faut bien gérer les enfants » et selon E5 « si on fait ce métier, c'est parce qu'il y a des enfants qu'on doit aider dans tout leur parcours, de la maternelle jusqu'en $6^{\mathrm{e}}$ année, donc je pense que les interactions avec les enfants, c'est la première, c'est la priorité. » Pour ce qui est des interactions avec les parents et la direction, puisque cela se passe bien dans l'école où a eu lieu la recherche, les sujets nous ont indiqué qu'il ne s’agissait pas d'une priorité.

\section{Discussion}

Les résultats provenant du questionnaire, des observations et des entrevues mettent de l'avant un élément important concernant la perception du personnel du SGMS qui a participé à cette recherche en ce qui concerne la compétence à communiquer oralement. «S'exprimer clairement et correctement à l'oral » relèverait davantage d'une bonne compréhension générale de la part des élèves aux propos émis par le personnel du SGMS qu'au respect des règles grammaticales de la langue et à l'utilisation d'un registre de langue standard. Autrement dit, pour le personnel de ce SGMS, "s'exprimer clairement et correctement à l'oral » semble davantage en lien avec la capacité à transmettre un message plutôt qu'en lien avec la manière de dire ce message.

Aux yeux des répondants de la présente recherche, les enseignants seraient ceux qui auraient le plus d'influence sur la communication orale des enfants, suivi des parents. Ceux qui auraient le moins d'influence seraient les surveillants du diner, suivis par la direction. Malgré que les membres du personnel du SGMS de cette recherche considèrent en majorité être des modèles, ils ne se positionnent pas comme ceux qui ont le plus d'influence sur la communication orale des élèves (en quatrième position, juste après les autres enfants, pour les éducateurs et en sixième position pour les surveillants du diner). Le fait d'être en milieu plurilingue pourrait expliquer pourquoi les enseignants arrivent en première position. Puisque les enfants sont peu exposés au français à la maison, l'enseignant devient souvent la première référence pour les élèves. De plus, le fait que la majorité du personnel n’a pas le français comme langue première (4 personnes sur 12 ont le français comme langue première) pourrait expliquer pourquoi il ne se positionne pas parmi les gens qui ont le plus d'influence sur la communication orale malgré le fait que ce personnel reconnait son rôle de modèle linguistique. D’ailleurs, nous avons pu constater que le rôle de modèle linguistique, pour les sujets, concerne aussi l'utilisation de la langue française plutôt qu'une autre langue auprès des enfants. Il serait intéressant de comparer ces résultats dans un milieu où le français est plus homogène, tant chez les enfants que chez le personnel du SGMS.

Les observations que nous avons menées pendant huit jours ont été déterminantes dans le cadre de cette recherche pour bien comprendre le travail effectué par le personnel du SGMS qui a participé à la recherche, mais aussi pour comprendre les réponses du questionnaire et pour préparer les entrevues. Sans ces observations, plusieurs résultats de cette recherche auraient été absents, incomplets ou difficilement analysables. Un élément 
qui apparaissait peu dans le questionnaire et les entrevues, mais de façon flagrante lors de nos observations est la relation directe entre la gestion du groupe et la compétence à communiquer oralement du personnel que nous avons observé. En effet, à plusieurs reprises, nous avons pu constater que des interventions auprès des enfants, et aussi auprès des parents et des collègues, étaient réussies en raison de stratégies de communication orale mises en place par les sujets. Ces stratégies avaient une incidence directe sur la gestion du groupe et sur la relation avec les adultes (parents, éducateurs, etc.). Nous avons pu observer des reformulations faites par les sujets ou demandées aux enfants, des demandes de confirmation pour vérifier la compréhension, l'utilisation de la rétroaction sandwich, l'utilisation de faits plutôt que d'impressions pour discuter d'une situation, le fait pour la personne de se mettre au niveau de l'enfant pour communiquer avec lui plutôt que de le regarder de haut, l'utilisation du non-verbal, la position du sujet dans l'espace pour observer ou intervenir, etc. (De Vito, 1993; Dumais, 2011; Berthiaume, 2016; Lavédrine, 2016). De nouvelles observations, cette fois filmées, pourraient être effectuées afin d'amener le personnel des SGMS à s'observer et à expliquer, s'ils en sont conscients, les stratégies qu'ils utilisent. Des entretiens d'autoconfrontation pourraient être effectués afin de mieux comprendre les stratégies ainsi mises en place par le personnel (Cadet, 2014). Ces données pourraient aider à la formation continue du personnel des SGMS.

Dans le questionnaire, nous n'avons pas abordé la question de la collaboration entre les enseignants et le personnel du SGMS. Toutefois, à partir de nos observations, nous avons cru percevoir un problème à ce sujet. Les entrevues que nous avons menées ont confirmé nos observations. Nos résultats de recherche appuient d'autres recherches qui ont soulevé ce problème important qu'est le manque de collaboration et de communication entre le personnel des SGMS et les enseignants, ce qui a des répercussions tant auprès des élèves que dans le travail même du personnel des SGMS (Levasseur \& Tardif, 2005; Chalifoux et al., 2014; Sorel Étienne et al., 2015; Tessier et al., 2016). Une réflexion devrait être entamée par les directions d'établissements scolaires et les commissions scolaires afin de déterminer des moments de rencontres entre tous les membres du personnel scolaire, incluant le personnel des SGMS. Ceci est en adéquation avec les recommandations du CSÉ (2017) qui souhaite mettre à profit le regard de tous les adultes qui côtoient les enfants dans les différents contextes de l'école afin d'améliorer les pratiques.

Enfin, nous ne pouvons passer sous silence les besoins en formation continue du personnel des SGMS. Les résultats que nous avons obtenus dans cette recherche, tout comme d'autres recherches (Giguère \& Desrosiers, 2010; Myre-Bisaillon \& Chalifoux, 2013; Caron, 2016), font état d'un besoin de formation continue pour le personnel des SGMS. Cette recherche permet d'avoir des données nouvelles quant à la compétence à communiquer oralement du personnel en SGMS et offre des pistes pour la formation initiale et continue, un besoin déjà mentionné dans la recherche de Myre-Bisaillon et Chalifoux (2013). La recherche que nous avons menée nous laisse croire que la formation continue doit se faire dans le milieu de travail du personnel des SGMS afin de pouvoir identifier des pratiques dites efficaces qui sont déjà en place, qui répondent aux besoins réels du milieu et qui serviront de références. Il nous est possible de croire que les besoins de formation ne seront pas les mêmes en milieu défavorisé, plurilingue et pluriethnique que dans un milieu plus francophone et plus favorisé, notamment en ce qui concerne la 
compétence à communiquer oralement, d’où la nécessité de former le personnel dans son milieu.

\section{Conclusion}

Cette recherche exploratoire est l'une des premières à documenter précisément les perceptions de la compétence à communiquer oralement du personnel d'un SGMS. Nous sommes cependant conscients du nombre restreint de participants et qu'il est impossible de généraliser les résultats, notamment en raison d’un milieu spécifique (défavorisé, plurilingue et pluriethnique). De plus, les observations effectuées sont limitées et ont été effectuées sur quatre sujets seulement. Les membres du personnel du SGMS qui avaient le plus de difficulté avec la compétence à communiquer oralement n’ont pas accepté de participer à la recherche ou se sont limités au questionnaire ou aux entrevues. Malgré cela, cette recherche ouvre la porte à plusieurs autres recherches en plus d'apporter des données scientifiques pour améliorer la formation initiale et continue du personnel des SGMS et, par le fait même, les compétences en littératie de ce personnel. Un premier pas dans cette direction a déjà été effectué avec la création d'une ressource pour aider le personnel des SGMS à mieux communiquer avec les enfants, les collègues et les parents (Dumais \& Soucy, 2016). Cette ressource présente six stratégies pour favoriser une communication orale réussie issues de nos observations auprès de quatre participants de cette recherche et de données provenant de recherches sur le sujet (voir l'annexe 1). Il serait maintenant intéressant de comparer les résultats de cette recherche avec d'autres SGMS afin de déterminer si les résultats sont un cas unique ou s'ils sont similaires dans l'ensemble du Québec et même dans les autres provinces canadiennes. Il parait également essentiel de s’intéresser aux répercussions de la communication orale dans la gestion de groupe dans les SGMS.

\section{Références}

Allen, N. (2014). L’oral réflexif au service du développement de la littératie volet oral au primaire : regard sur la compréhension orale. Language and Literacy, 16(2), 1-16. Repéré à

https://journals.library.ualberta.ca/langandlit/index.php/langandlit/article/viewFile/ $\underline{21264 / 18127}$

Association des services de garde en milieu scolaire du Québec (2011). Promouvoir, soutenir, représenter. Rapport annuel 2010-2011. Repéré à http://www.gardescolaire.org/aqgs/rapport-annuel/

Baillargeon, M., Caouette, C.É., \& Darveau, P. (1990). Rôle social et éducatif du service de garde en milieu scolaire tiré des actes du $4^{e}$ congrès sur les services de garde en milieu scolaire «Grandir avec toi ». Québec : Bibliothèque nationale du Québec.

Bardin, L. (2009). L'analyse de contenu. Paris : Presses universitaires de France.

Berger, D., Héroux, D., \& Shéridan, D. (2012). L'éducation à l'enfance. Une voie professionnelle à découvrir ( $2^{\mathrm{e}}$ édition). Montréal : Gaëtan Morin éditeur.

Bergeron, R., Dumais, C., Harvey, B., \& Nolin, R. (2015). La didactique du français oral du primaire à l'université. Côte Saint-Luc : Éditions Peisaj. Repéré à http://peisaj.ca/La_didactique_du_fran\%C3\%A7ais_oral_du_primaire_\%C3\%A0_l universit\%C3\%A9.php 
Berthiaume, D. (2016). L'observation de l'enfant en milieu éducatif (2édition). Montréal : Chenelière éducation.

Cadet, L. (2014). Corps de l'enseignant : sujet de la recherche, objet de la formation, in : M. Tellier \& L. Cadet (Eds.), Le corps et la voix de l'enseignant : théorie et pratique (pp. 189-206). Paris : Éditions maison des langues.

Caron, P. (2016). Le travail des éducatrices en garde scolaire. Mémoire de maitrise, Université de Montréal. Repéré à https://papyrus.bib.umontreal.ca/xmlui/handle/1866/18605

Cayouette, M. (2017). Ce n'est pas mon local, c'est celui des élèves. Nouvelles CSQ, printemps, 17.

Chalifoux, A., Myre-Bisaillon, J., \& Bergeron, J. (2014). Un projet d'éveil à la lecture et à l'écriture dans les services de garde en milieu scolaire. Vivre le primaire, 27(4), 44-46.

Cloutier, S. (2012). L'étayage. Agir comme guide pour soutenir l'autonomie. Pour un enfant à son plein potentiel. Québec : Presses de l’Université du Québec.

Conseil de la famille et de l'enfance (2006). Les familles, l'école et son service de garde : un projet éducatif partagé? Québec : Gouvernement du Québec. Repéré à https://www.mfa.gouv.qc.ca/fr/publication/documents/cfe_etude_projet_educatif_p artage.pdf

Conseil supérieur de l'éducation [CSÉ] (1996). Pour un développement intégré des services éducatifs à la petite enfance : de la vision à l'action. Québec : Gouvernement du Québec. Repéré à https://www.cse.gouv.qc.ca/fichiers/documents/publications/50-0408.pdf

Conseil supérieur de l'éducation [CSÉ] (2006). Les services de garde en milieu scolaire : inscrire la qualité au cœur des priorités. Québec : Gouvernement du Québec. Repéré à https://www.cse.gouv.qc.ca/fichiers/documents/publications/Avis/500452.pdf

Conseil supérieur de l'éducation [CSÉ] (2012). Mieux accueillir et éduquer les enfants d'âge préscolaire, une triple question d'accès, de qualité et de continuité des services. Québec : Gouvernement du Québec. Repéré à https://www.cse.gouv.qc.ca/fichiers/documents/publications/Avis/50-0477.pdf

Conseil supérieur de l'éducation [CSÉ] (2017). Pour une école riche de tous ses élèves. S'adapter à la diversité des élèves, de la maternelle à la $5^{e}$ année du secondaire. Québec : Gouvernement du Québec. Repéré à http://www.cse.gouv.qc.ca/fichiers/documents/publications/Avis/50-0500.pdf

De Vito, J.A. (1993). Les fondements de la communication humaine. Boucherville : Gaëtan Morin éditeur.

Dumais, C. (2011). L'évaluation de l'oral par les pairs : pour une inclusion réussie de tous les élèves. Vie pédagogique, 158, 59-60. Repéré à

http://www.christiandumais.info/wpcontent/uploads/2008/01/L\%C3\%A9valuation-de-loral-par-les-pairs-Viep\%C3\%A9dagogique.pdf

Dumais, C. (2015). Les fondements d'une taxonomie du développement de la langue orale : un modèle pour développer les compétences à l'oral et en littératie des élèves de 6 à 17 ans, in : L. Lafontaine \& J. Pharand (Eds.), Littératie : vers une 
maitrise des compétences dans divers environnements (pp. 87-111). Québec : Presses de l’Université du Québec.

Dumais, C., \& Soucy, E. (2016). Stratégies pour mieux communiquer avec les enfants, les collègues et les parents. Revue Gardàvue de l'Association québécoise de la garde scolaire, 31(1), 22-23. Repéré à

https://www.dropbox.com/s/a2pnq261n23pdgo/Mieux\%20communiquer\%20article $\% 20$ publie.pdf?dl=0

Dumais, C., Soucy, E., \& Plessis-Bélair, G. (2017). La didactique de l’oral au préscolaire et au primaire au Québec : portrait d'un domaine de recherche en émergence, in S.

El Euch, A. Groleau \& G. Samson (Eds.), Les didactiques : bilans et perspectives (pp. 101-124). Québec : Presses de l’Université du Québec.

Fortin, M.-F., \& Gagnon, J. (2016). Fondements et étapes du processus de recherche. Méthodes quantitatives et qualitatives. Montréal : Chenelière éducation.

Gaudreau, L. (2011). Guide pratique pour créer et évaluer une recherche scientifique en éducation. Montréal : Guérin.

Giguère, C., \& Desrosiers, H. (2010). Les milieux de garde de la naissance à 8 ans : utilisation et effets sur le développement des enfants. Québec : Institut de la statistique du Québec. Repéré à http://www.stat.gouv.qc.ca/statistiques/education/milieu-garde/milieux-garde.pdf

Gouvernement du Québec (2017). Règlement sur les services de garde en milieu scolaire. Québec : Éditeur officiel du Québec. Repéré à http://legisquebec.gouv.qc.ca/fr/pdf/cr/I-13.3,\%20R.\%2011.pdf

Lacelle, N., Lafontaine, L., Moreau, A.C., \& Laroui, R. (2016). Définition de la littératie. Réseau québécois de recherche et de transfert en littératie. Repéré à http://www.ctreq.qc.ca/realisation/reseau-quebecois-sur-la-litteratie/

Lafontaine, L., \& Pharand, J. (2015). La littératie. Un concept en évolution, in : L. Lafontaine \& J. Pharand (Eds.), Littératie : vers une maitrise des compétences dans divers environnements (pp. 1-11). Québec : Presses de l’Université du Québec.

Lavédrine, C. (2016). Assumer son autorité et motiver sa classe. Techniques vocales, corporelles et verbales pour entrainer, captiver et transmettre. Louvain-la-Neuve : De Boeck supérieur.

L’Écuyer, R. (1987). L’analyse de contenu: Notion et étapes, in : J.-P. Deslauriers (Eds.), La recherche qualitative : Résurgence et convergences (pp. 49-66). Ste-Foy, Québec : Presses de l’Université du Québec.

Levasseur, L., \& Tardif, M. (2005). Les rapports professionnels entre les techniciens et les enseignants dans la division du travail au Québec. Éducation et sociétés, 1(15), 169-188. Repéré à http://www.cairn.info/revue-education-et-societes-2005-1-page169.htm

Manningham, S. (2008). Qualité de l'environnement éducatif dans les services de garde préscolaires au Québec : rôle des caractéristiques de l'éducatrice et une intervention visant à augmenter ses compétences. Thèse de doctorat, Université de Montréal. Repéré à https://papyrus.bib.umontreal.ca/xmlui/handle/1866/6380

Messier, G., Dumais, C., \& Viola, S. (2012). Portrait des perceptions de la communication orale des étudiants de première année en formation des maitres, in : R. Bergeron \& G. Plessis-Bélair (Eds.), Représentations, analyses et descriptions 
du français oral, de son utilisation et de son enseignement au primaire, au secondaire et à l'université (pp. 97 à 112). Côte St-Luc : Éditions Peisaj.

Miles, M. B., \& Huberman, A. M. (2003). Analyse des données qualitatives ( ${ }^{\mathrm{e}}$ édition). Paris : De Boeck Université.

Ministère de l'Éducation du Québec (2001). Programme de formation de l'école québécoise, éducation préscolaire, enseignement primaire. Québec :

Gouvernement du Québec. Repéré à

http://www.education.gouv.qc.ca/fileadmin/site_web/documents/PFEQ/prform2001 . pdf

Mottet, M. (2009). Parler en bon français. Quelle représentation les futurs enseignants du primaire en ont-ils? Québec français, 155, 89-91.

Mottet, M., \& Gervais, F. (2007). Représentations et réactions affectives de futurs enseignants à l'égard du français québécois oral soigné, de la culture et de la didactique de l'oral. Revue des sciences de l'éducation, 33(2), 339-356.

Myre-Bisaillon, J., \& Chalifoux, A. (2013). Les perceptions du personnel des services de garde en milieu scolaire défavorisé dans le cadre d'un projet d'éveil à la lecture et à l'écriture auprès d'élèves de maternelle. Canadian Journal of Education, 36(4), 115-143. Repéré à http://journals.sfu.ca/cje/index.php/cje-rce/article/view/1367

Myre-Bisaillon, J., Chalifoux, A., Lapointe-Garant, M., Dionne, C., \& Rodrigue, A. (2014). Éveil à la lecture et à l'écriture dans les services de garde en milieu scolaire : engagement et ouverture face aux livres. Nouveaux cahiers de la recherche en éducation, 17(2), 125-147. Repéré à https://www.erudit.org/fr/revues/ncre/2014-v17-n2-ncre01902/1030890ar.pdf

Sauvé, L. (1992). Éléments d'une théorie du design pédagogique en éducation relative à l'environnement : élaboration d'un supramodèle pédagogique. Thèse de doctorat inédite, Université du Québec à Montréal.

Savoie-Zajc, L. (2011). La recherche qualitative/interprétative en éducation, in : T. Karsenti \& L. Savoie Zajc (Eds.), La recherche en éducation : étapes et approches (3e édition) (pp. 123-147). Saint-Laurent : ÉRPI.

Sorel Étienne, L., Lambert Deubelbeiss, C., \& Rousseau, A. (2015). L'éthos du personnel éducateur des services de garde en milieu scolaire. Rapport de recherche présenté à l'Association des services de garde en milieu scolaire du Québec. Québec : Université Laval. Repéré à http://www.cms.fss.ulaval.ca/upload/soc/fichiers/lab_rech_2015_rpt_final_asgems q.pdf

Soucy, E. (2016).Quelle place pour l'oral dans les centres de littératie?. Language and Literacy, 18(2), 1-16. Repéré à https://journals.library.ualberta.ca/langandlit/index.php/langandlit/article/view/2836 $\underline{0}$

Soucy, E., \& Dumais, C. (2017). Quelle place pour le personnel du service de garde en milieu scolaire dans les écoles primaires?. Vivre le primaire, 30(1), 26-27.

Tessier, O., Turcotte, S., Perreault, G., Beaudoin, S., \& Berrigan, F. (2016). La cour d'école : un lieu à privilégier pour favoriser le développement des élèves. Vivre le primaire, 24(1), 26-28. 


\section{Biographie des auteurs}

Christian Dumais est professeur de didactique du français à l'Université du Québec à Trois-Rivières. Ses recherches portent principalement sur l'enseignement et l'évaluation de l'oral du préscolaire à la fin du secondaire ainsi que sur la formation des maitres en ce qui concerne la compétence à communiquer oralement. Ses champs d'intérêt professionnels concernent également le développement des compétences en littératie, la compétence à communiquer oralement en service de garde en milieu scolaire et l'enseignement de l'orthographe lexicale. Il est chercheur régulier de l'Équipe de recherche en littératie et inclusion (ÉRLI) en plus de faire partie du Centre de recherche interuniversitaire sur la formation et la profession enseignante (CRIFPE) et d'être coresponsable du Laboratoire universitaire de recherche et de formation en didactique du français (LUDIF) de l'UQTR.

Emmanuelle Soucy est doctorante en science de l'éducation à l'Université du Québec en Outaouais. Ses intérêts de recherche portent sur la didactique du français au primaire. Dans le cadre de sa thèse, elle s'intéresse particulièrement aux centres de littératie et à l'enseignement de l'oral. Elle est enseignante au primaire depuis plusieurs années et a œuvré à titre d'enseignante-conseil, chargée de soutenir l'implantation de centres de littératie. Elle est également chargée de cours, superviseure de stage et assistante de recherche.

Annexe 1 : Six stratégies pour mieux communiquer avec les enfants, les collègues et les parents (Dumais et Soucy, 2016)

\section{Six stratégies pour mieux communiquer}

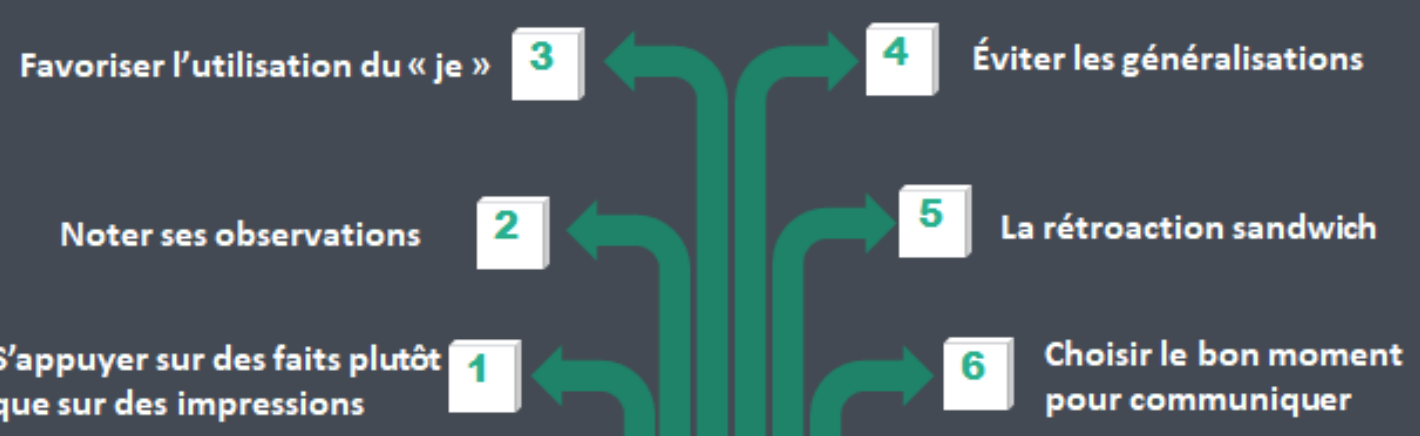
que sur des impressions pour communiquer 